\title{
Regulation of REG4 Expression and Prediction of 5-Fluorouracil Sensitivity by CDX2 in Ovarian Mucinous Carcinoma
}

\author{
IEMASA KOH, SUGURU NOSAKA, MASAKI SEKINE, JUN SUGIMOTO, EIJI HIRATA and YOSHIKI KUDO \\ Department of Obstetrics and Gynecology, Faculty of Medicine Graduate School of \\ Biomedical and Health Sciences, Hiroshima University, Hiroshima, Japan
}

\begin{abstract}
Background/Aim: The biological importance of the caudal-related homeobox transcription factor CDX2 in acquiring resistance to anticancer drugs has been studied in ovarian mucinous carcinoma. CDX2 promotes the expression of multidrug resistance 1 (MDR1) and confers resistance to paclitaxel. The regenerating islet-derived family member 4 (REG4) gene is a potential target gene of CDX2. In this study, we investigated the relationship between the expression of CDX2 and Reg IV and the regulation of Reg $I V$ expression and examined novel chemotherapeutic regimens. Materials and Methods: The regulation of Reg IV expression by CDX2 and sensitivity of 5-fluorouracil (5-FU) were evaluated using ovarian mucinous cancer cell lines. Results: The correlation of CDX2 with Reg IV expression was demonstrated in ovarian mucinous carcinoma. Reg IV expression was enhanced by transfection of CDX2 and was suppressed by inhibition of CDX2 expression. OMC-3 cells with ectopically overexpressed CDX2 showed enhanced apoptosis and sensitivity to 5-FU. Conclusion: CDX2 promotes resistance to paclitaxel and sensitivity to 5-FU. Novel 5-FU-based chemotherapy based on CDX2 may be used in ovarian mucinous carcinoma.
\end{abstract}

Ovarian cancer has the worst prognosis among gynecological malignant tumors. Ovarian cancer is often found as advanced cancer, because there is no effective method for early

This article is freely accessible online.

Correspondence to: Iemasa Koh, Department of Obstetrics and Gynecology, Faculty of Medicine Graduate School of Biomedical and Health Sciences, Hiroshima University, 1-2-3, Kasumi, Minami-ku, Hiroshima 734-8551, Japan. Tel: +81 822575262, Fax: +81 822575264, e-mail: iemasakoh550317@gmail.com

Key Words: CDX2, 5-fluorouracil, REG4, mucinous carcinoma, ovarian cancer. diagnosis. Treatment of epithelial ovarian cancers (EOCs) involves a combination of chemotherapy and surgery, however, acquired resistance to anticancer drugs is very common. With the discovery of novel biomarkers and target genes for diagnosis and treatment, it is thought that a better understanding of gene expression changes and the related molecular mechanisms occurring in the carcinogenesis of ovarian cancer may lead to improvements in diagnosis, treatment and prevention of ovarian cancer.

Ovarian mucinous cancer is a histological type that accounts for approximately $10 \%$ of EOCs (1). Currently, the standard chemotherapy regimen for initial treatment of EOCs is a combination of carboplatin and paclitaxel, but ovarian mucinous carcinoma is known to be resistant to this regimen (2-6). Ovarian mucinous carcinoma is often diagnosed at an early stage, but apparently has a poorer prognosis than other histological types (2-4). The expression of multidrug resistance 1 (MDR1), also known as the multidrug resistance gene, causes drug resistance. In our previous study, it was suggested that the expression of MDR1 was regulated by the intestine-specific caudal-related homeobox transcription factor, $\mathrm{CDX} 2$, and was related to drug resistance in ovarian mucinous carcinoma and colorectal cancer $(7,8)$. In addition, it has been reported that 5-fluorouracil (5-FU), a key drug used in the treatment of colon cancer, is also effective against ovarian mucinous cancer (9). CDX2 has been shown to have an important role in intestinal development and differentiation and in the maintenance of the intestinal phenotype $(10,11,12)$. It has also been involved in the degree of differentiation, malignant grade and carcinogenesis in gastric and colorectal cancer $(10,13,14)$. A potential novel chemotherapy regimen based on the expression kinetics of CDX2 against ovarian mucinous carcinoma has been suggested (7).

In addition, it has been recently reported that the expression of Reg IV is directly regulated by CDX2 in gastric cancer (GC) (15). Regenerating islet-derived family member 4 (REG4, which encodes Reg IV protein) is a member of the 
Table I. Real-time PCR primers.

\begin{tabular}{lll}
\hline Gene name & Forward primer $5^{\prime} \rightarrow 3^{\prime}$ & Reverse primer $5^{\prime} \rightarrow 3^{\prime}$ \\
\hline CDX2 & GAACCTGTGCGAGTGGATG & GGTGATGTAGCGACTGTAGTGAA \\
$R E G 4$ & GCCCCGCCATCCCTT & CTGCTCGAGACAGCCAGAGA \\
\hline
\end{tabular}

$R E G$ gene family. The REG gene family belongs to the calcium-dependent lectin superfamily $(15,16)$. REG4 expression is closely linked to the differentiation of ovarian mucinous carcinoma. It has also recently been reported that staging of the International Federation of Gynecology and Obstetrics (FIGO), differentiation and the expression of Reg IV protein is an independent predictor of overall survival in ovarian cancer and that Reg IV expression is a predictor of progression-free survival (17). Reg IV is a potent activator of the epidermal growth factor receptor (EGFR)/Akt/activating protein-1 (AP-1) signaling pathway in colon cancer cells. Reg IV increases the expression of Bcl-2 and leads to inhibition of apoptosis $(15,18)$. In gastric cancer cells, it has been shown to inhibit apoptosis induced by 5-FU through activating EGFR (19). REG4 has also been identified as one of the genes involved in the development of cancer (20).

In ovarian cancer, the relationship between CDX2 and Reg IV is not known. Therefore, the expression of CDX2 and Reg IV in clinical tissues of EOCs was investigated. In addition, regulation of Reg IV by $\mathrm{CDX} 2$, activation of EGFR/Akt/AP-1 signaling pathway by Reg IV, and sensitivity to5-FU were also evaluated using ovarian mucinous cancer cell lines.

\section{Materials and Methods}

Immunohistochemical staining in clinical tissues. We investigated 22 cases of EOCs diagnosed and resected at our institution between 2010 and 2013. Formalin-fixed, paraffin-embedded (FFPE) tissues were stained by the avidin-biotin complex method using rabbit monoclonal anti-CDX2 antibodies (clone EPR2764Y; NICHIREI, Tokyo, Japan) and mouse monoclonal anti-REG4 antibodies (clone AF1379; R\&D, Minneapolis, MN, USA), as previously described $(7,14)$. Antibodies were used at 1:1 and 1:50 dilution, respectively. Tissue specimens were collected and used according to the Ethical Guidelines for Human Genome/Gene Research enacted by the Japanese Government.

Cell lines, retrovirus infections, and cell culture. The five cell lines of ovarian mucinous carcinoma used in a previous study were also used in this study (MCAS, RMUG-S, MN-1, OMC-1 and OMC-3) (7). We obtained these cell lines from the following sources: $\mathrm{MN}-1$ from Scienstuff Co. Ltd (Nara, Japan) (21), OMC-1 from Dr. Tsuyoshi Saito (School of Medicine, Sapporo Medical University, Sapporo, Japan) (22), MCAS and RMUG-S from the Japanese Collection of Research Bioresources (JCRB) Cell Bank, and OMC-
3 from RIKEN Bio Resource Center Cell Bank (Institute of Physical and Chemical Research, Tsukuba, Japan). Culture conditions used for the five cell lines, have been previously described (7).

The pPGS-CDX2 was generated by inserting the $C D X 2$ gene in the retroviral expression vector pPGS-CMV-CITE-neo (provided by G. Nabal, NIH, Bethesda, MD, USA). As previously described (7, 14), OMC-3/PGS-CDX2 was generated by transfecting CDX2 into OMC-3 cells with low expression of endogenous CDX2, and control cells (OMC-3/PGS-neo) were also generated. The expression of CDX2 in OMC-3/PGS-CDX2 and OMC-3/PGS-neo cells was examined using real time PCR and western blot.

Quantitative real-time reverse transcription PCR. As described in the manufacturer's protocol, total RNA was isolated by the RNeasy Mini Kit (Qiagen, Valencia, CA, USA) and reverse transcribed by the Omniscript RT kit (Qiagen). One $\mu$ g of total RNA was used for cDNA synthesis. Real-time reverse transcription PCR (RT-qPCR) was performed using sequence-specific primers indicated in Table I and Power SYBR Green PCR Master Mix (Applied Biosystems, Foster, CA, USA) on an ABI 7300HT.

Western blot analysis. Western blot analysis was performed as described previously $(7,14)$. Anti-CDX2 mouse monoclonal (clone CDX2-88; BioGenex Laboratories, Inc. Fremont, CA, USA), and anti-human Reg IV monoclonal antibody (Proteintech, USA) were used at 1:100, and 1:50 dilutions, respectively. Anti-EGFR antibody (clone D38B1; Cell Signaling Technology, Beverly, MA, USA), anti-phospho-EGFR (Tyr992) antibody (Cell Signaling Technology), anti-phospho-EGFR (Tyr1068) antibody (clone D7A5; Cell Signaling Technology), anti-Akt (pan) antibody (clone C67E7; Cell Signaling Technology), anti-phospho-Akt (Thr308) antibody (clone C31E5E; Cell Signaling Technology), anti-Bcl-2 antibody (clone 124; Santa Cruz Biotechnology, Santa Cruz, CA, USA), and antiDPD antibody (Taiho Pharmaceutical, Tokyo, Japan) were used to evaluate the effects on the EGFR/Akt/AP-1 pathway and apoptosis. Anti-GAPDH antibody (clone 6C5; Santa Cruz Biotechnology) was also detected as a loading control.

RNA interference. Silencing was performed using RNAiMAX (Invitrogen, Carlsbad, CA, USA) as described previously (7). Quantitative RT-qPCR was performed for validation. Two small interfering RNA (siRNA) duplexes targeting CDX2 mRNA (5'AACCAGGACGAAAGACAAAUA-3', CDX2 siRNA1; and 5'AAGCCUCAGUGUCUGGCUCUG-3', CDX2 siRNA2) and a nonsilencing siRNA duplex (MISSION siRNA Universal Negative Control SIC-001) were synthesized by Qiagen-Xeragon (Huntsville, AL, USA).

MTS and apoptosis assays. MTS cytotoxicity assay was performed using an ImmunoMini NJ-2300 microplate reader (InterMed, Tokyo, Japan) to examine cell survival following exposure to chemotherapeutic 


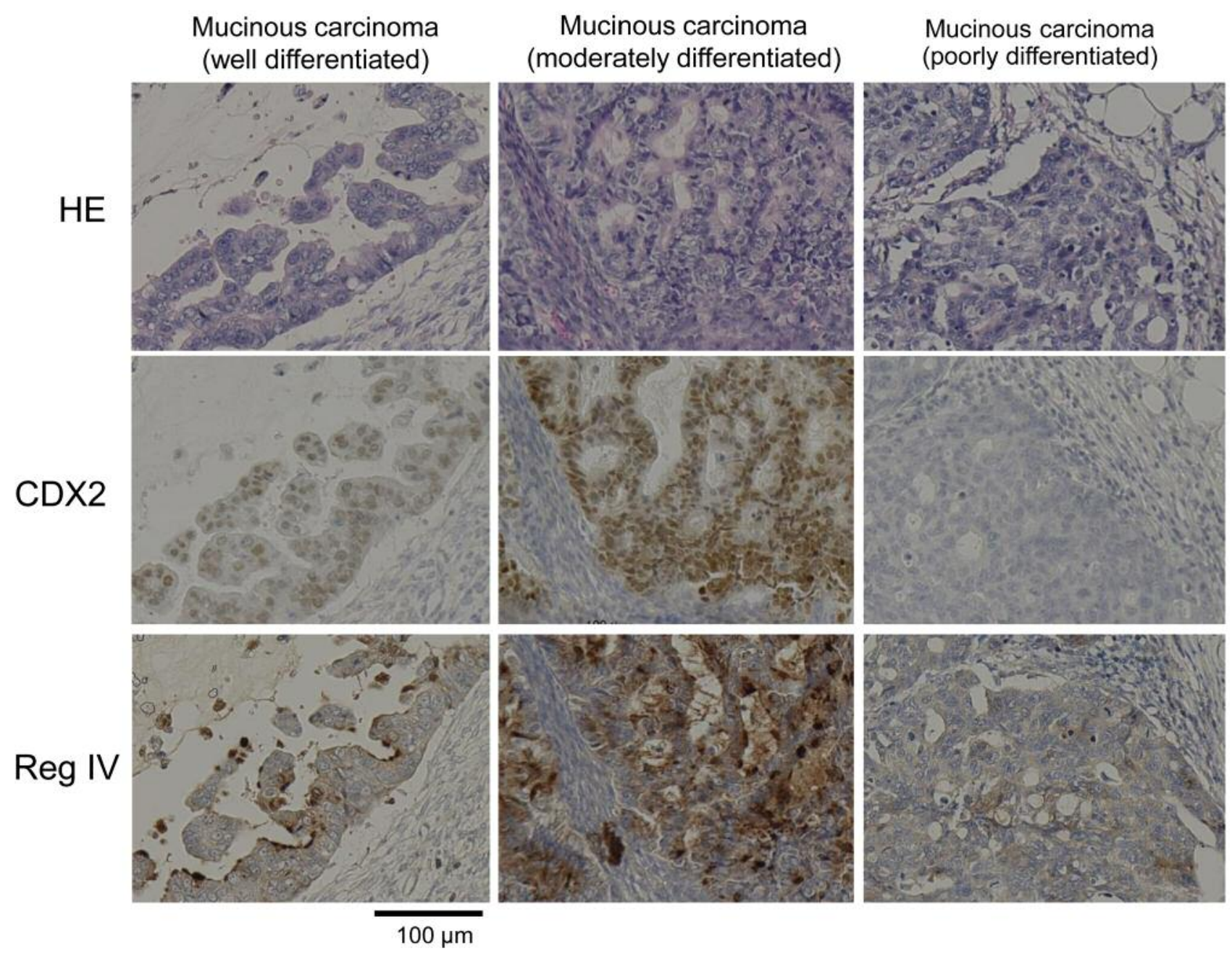

Figure 1. In ovarian mucinous carcinoma, the expression of CDX2 and Reg IV was correlated with the degree of differentiation of cancer cells. For immunohistochemical examination, formalin-fixed paraffin-embedded tissues were used. Hematoxylin and eosin staining (upper), anti-CDX2 monoclonal antibody (middle) and anti-Reg IV monoclonal antibody (lower) were used.

agents as described previously (7). 5-FU was obtained from Wako (Oosaka, Japan). Cells were seeded at 10000 cells/100 $\mu$ l per well in 96-well microtiter plates. The concentration required to inhibit cell growth by $50 \%$ relative to that of nontreated control cells [ $\left.\mathrm{IC}_{50}(96 \mathrm{~h})\right]$ was assessed.

Apoptotic assays were performed using a Cell Death Detection ELISAPlus Kit (Roche Diagnostics) to evaluate apoptosis following treatment of cells for $48 \mathrm{~h}$ with $5 \mathrm{mM} \mathrm{5-FU,} \mathrm{according} \mathrm{to} \mathrm{the}$ manufacturer's instructions.

\section{Results}

Co-expression of CDX2 and Reg IV in human ovarian cancer tissue. Immunohistochemical staining analysis revealed that the expression of Reg IV is localized in the membrane, whereas the expression of the CDX2 transcription factor is localized in the nucleus (Figure 1). Of the 22 cases of ovarian cancer, 5 were CDX2 expression positive $(22.7 \%)$ and Reg IV expression positive (22.7\%) (Table II). CDX2 expression was observed in well and moderately differentiated ovarian mucinous carcinoma, but expression was not observed in the poorly differentiated type. Reg IV expression was observed in all 5 cases $(100 \%)$ of CDX2 positive; Reg IV expression was not detected serous and clear cell carcinomas. These immunohistochemical findings indicated that Reg IV expression was associated with CDX2 expression in mucinous ovarian cancer.

Regulation of Reg IV expression by CDX2 in ovarian mucinous carcinoma cells. We examined the correlation between Reg IV and CDX2 expression in ovarian mucinous carcinoma cell 
A
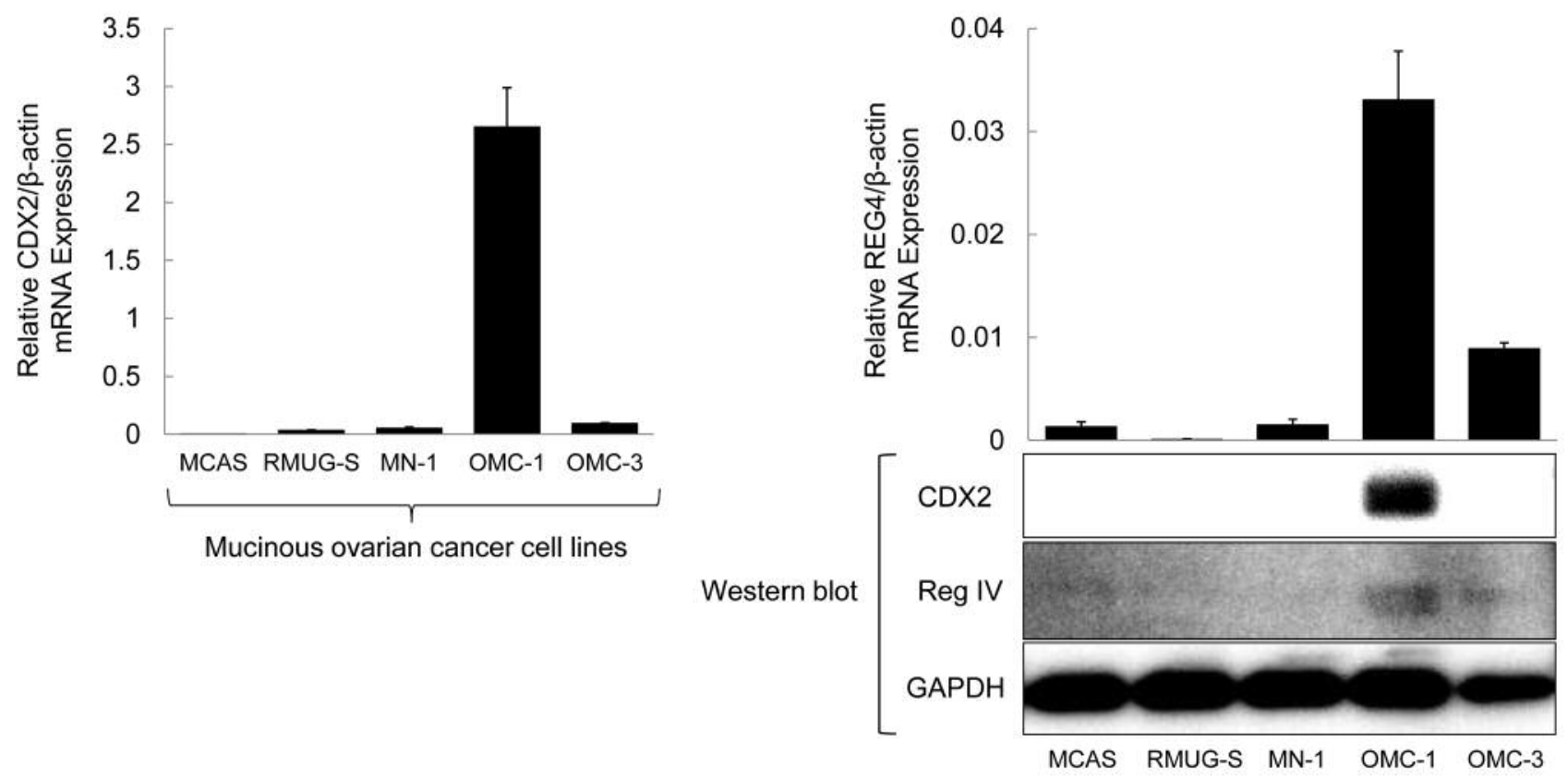

B
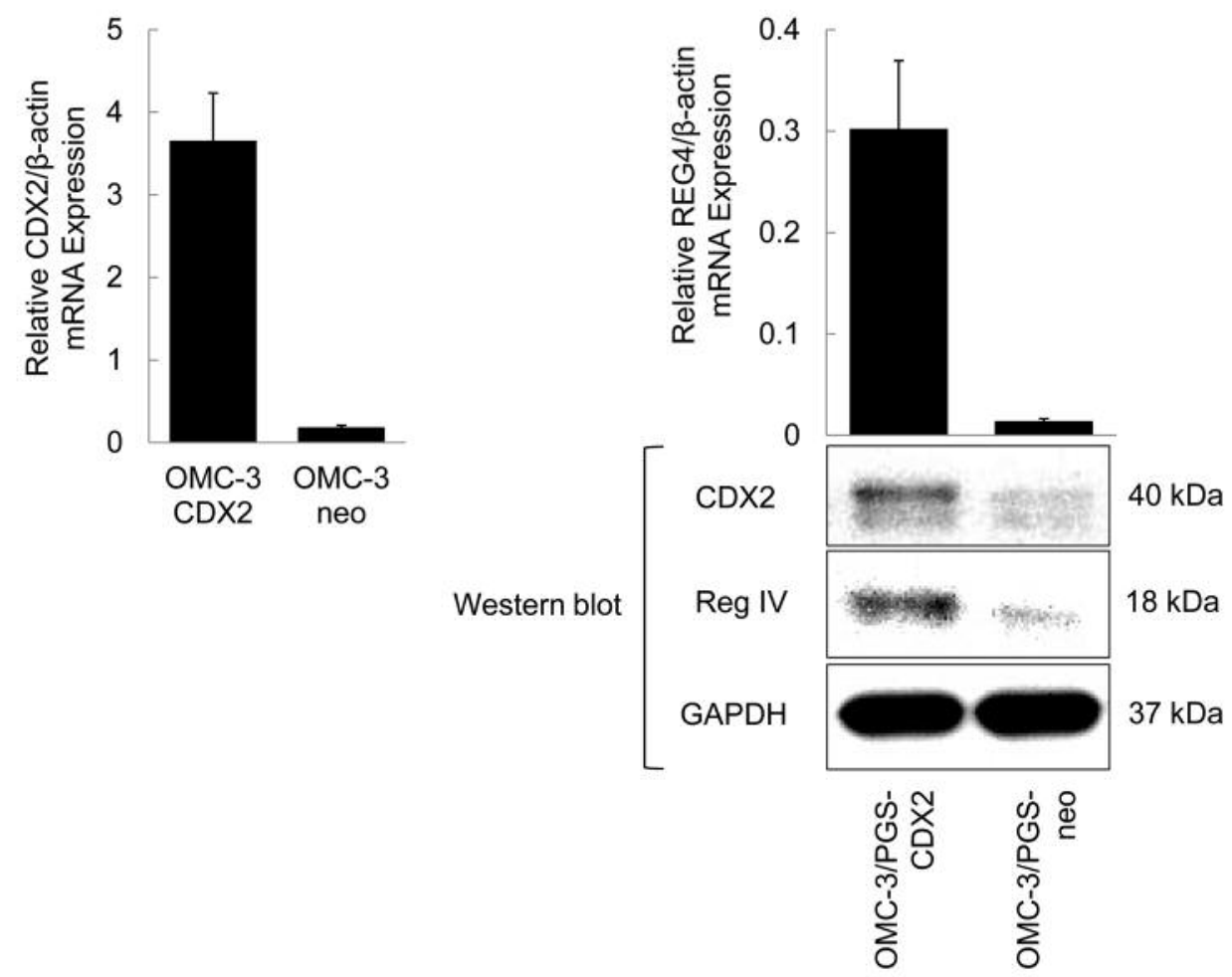

Figure 2. Expression of CDX2 and REG4 in five cell lines of ovarian mucinous carcinoma. (A) Expression of Reg IV and CDX2 was confirmed by western blot analysis, using anti-CDX2 (40 kDa), and anti-human Reg IV (18 kDa) monoclonal antibodies. Expression of relative CDX2/beta-actin and relative REG4/beta-actin $m R N A$ was confirmed by quantitative real-time (RT-qPCR) analysis, using CDX2 and REG4 primers, respectively. (B) OMC-3/PGS-CDX2 was generated by transfecting CDX2 into OMC-3 cells with low expression of endogenous CDX2. Control cells (OMC3/PGS-neo) were also generated. REG4 transcript and Reg IV protein were confirmed in OMC-3/PGS-CDX2 cells by RT-qPCR and western blotting, respectively. 

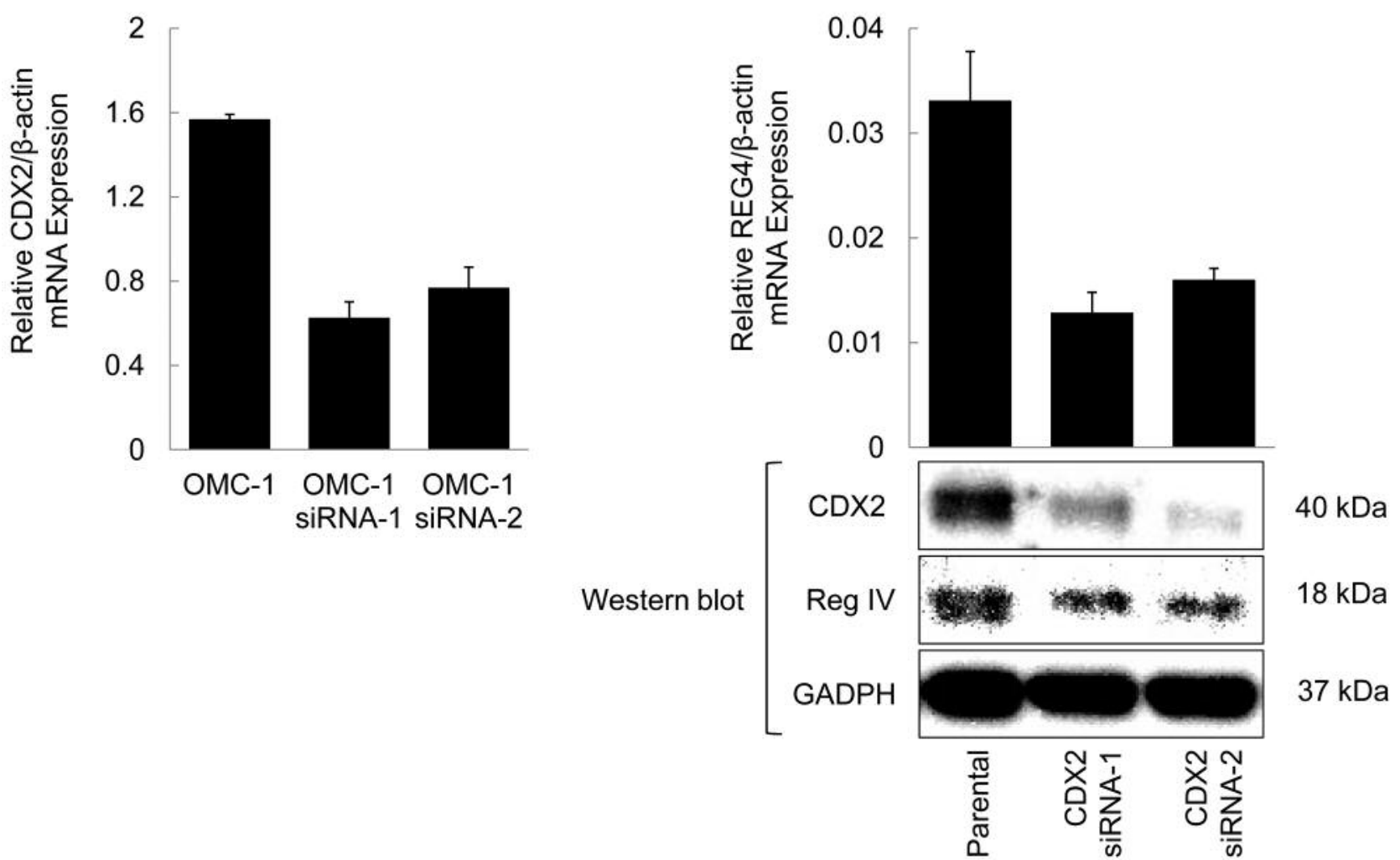

Figure 3. REG4 transcript and Reg IV protein levels in OMC-1 cells treated with CDX2 siRNAs were suppressed by approximately $50 \%$ compared with the levels observed in parental cells. Assays were performed in triplicate; columns, mean; bars, SD.

lines. High expression of REG4 and CDX2 mRNA and protein in OMC-1 cells was revealed by RT-qPCR and western blot analyses; expression of Reg IV and CDX2 was not observed in MCAS, RMUG-S, or MN-1 cells. In OMC- 3 cells, weak Reg IV expression was observed, but expression of CDX2 was not detected (Figure 2A). None of the cell lines without Reg IV expression displayed expression of CDX2.

To confirm whether CDX2 regulates REG4 expression in ovarian mucinous carcinoma cells, we generated OMC-3 cells with high levels of CDX2 expression (OMC-3/PGSCDX2 cells) by retroviral infection $(7,14)$. The expression of CDX2 was induced in OMC-3/PGS-CDX2 cells, but not in OMC-3/PGS-neo control cells. Induction of high expression of REG4 gene and Reg IV proteins in OMC3/PGS-CDX2 cells was confirmed by both RT-qPCR and western blot analysis (Figure 2B).

Down-regulation of REG4 expression by inhibition of CDX2 . To confirm the regulation of REG4 expression by CDX2 in ovarian mucinous carcinoma cells, CDX2 expression was suppressed by RNA interference (RNAi) and the effect on $R E G 4$ and Reg IV protein expression was evaluated. CDX2 protein expression was significantly suppressed by CDX2
Table II. Immunohistochemical staining in epithelial ovarian cancers.

\begin{tabular}{lcc}
\hline Histological type & CDX2 (\%) & Reg IV (\%) \\
\cline { 2 - 3 } Differentiation degree $(\mathrm{n}=22)$ & + & + \\
\hline Ovarian mucinous carcinoma $(\mathrm{n}=7)$ & & \\
$\quad$ Well-differentiated $(\mathrm{n}=3)$ & $3 / 3(100)$ & $3 / 3(100)$ \\
$\quad$ Moderately-differentiated $(\mathrm{n}=1)$ & $1 / 1(100)$ & $1 / 1(100)$ \\
Poorly-differentiated $(\mathrm{n}=3)$ & $0 / 3(0)$ & $0 / 3(0)$ \\
Ovarian serous carcinoma $(\mathrm{n}=5)$ & $0 / 5(0)$ & $0 / 5(0)$ \\
Ovarian endometrioid carcinoma $(\mathrm{n}=5)$ & $1 / 5(20)$ & $1 / 5(20)$ \\
Ovarian clear cell carcinoma $(\mathrm{n}=5)$ & $0 / 5(0)$ & $0 / 5(0)$ \\
\hline
\end{tabular}

Positive (+): $>50 \%$ of tumor cells stained; Well-differentiated: Ratio of solid growth part 0-5\%; Moderately-differentiated: 5-50\%; Poorlydifferentiated: $50-100 \%$.

specific siRNA in OMC-1 cells; REG4 transcript and Reg IV protein levels in OMC-1 cells treated with CDX2 siRNAs were down-regulated by approximately $50 \%$ compared with levels observed in parental cells (Figure 3). These data indicated that CDX2 regulates REG4 gene and Reg IV protein expression in $\mathrm{OMC}-1$ cells. 

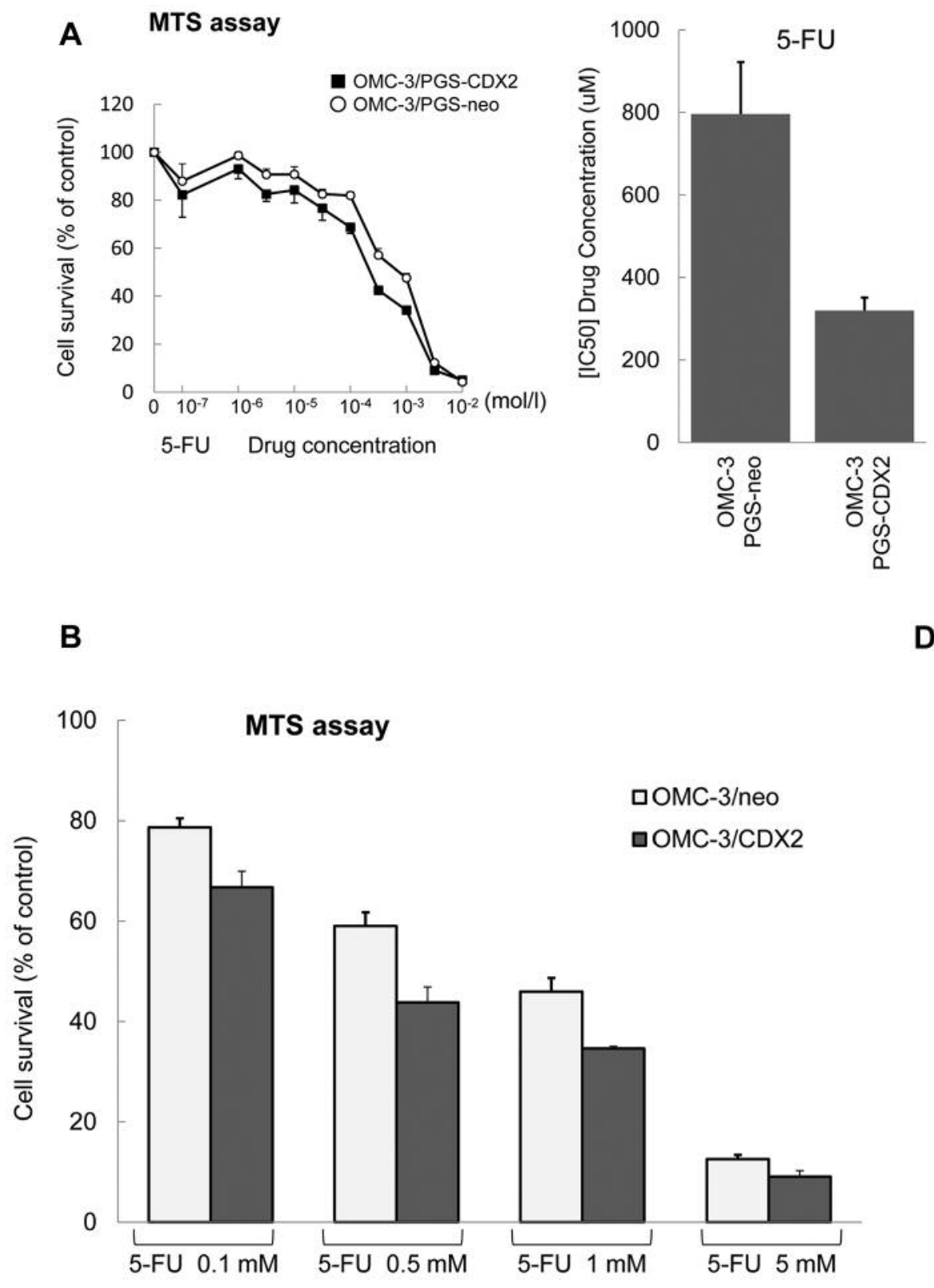
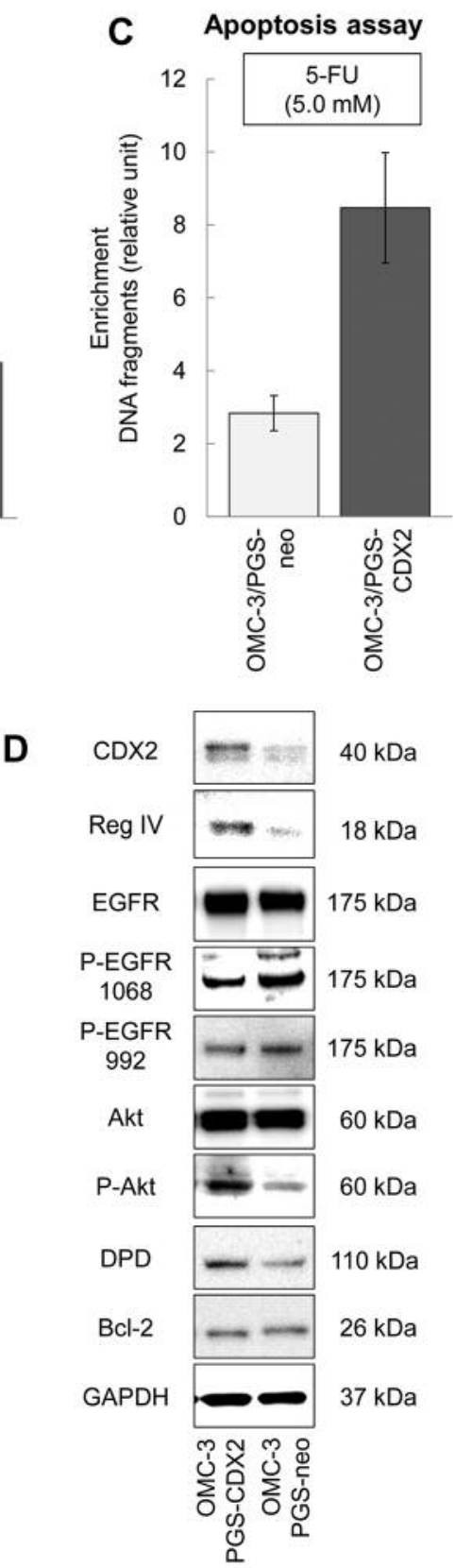

Figure 4. OMC-3 cells ectopically expressing CDX2 display sensitivity to 5-FU. (A) Effect of 5-FU treatment on OMC-3/PGS-CDX2 (ם) and OMC3/PGS-neo $(\bigcirc)$ cell lines. Increased cytotoxicity caused by 5-FU was observed in OMC-3/PGS-CDX2 compared to OMC-3/PGS-neo cells. (B) Cell growth of OMC-3/PGS-neo cells was inhibited by 5-FU in a dose-dependent manner. It was more strongly inhibited in OMC-3/PGS-CDX2 cells. (C) OMC-3/PGS-CDX2 cells significantly induced apoptosis in comparison with OMC-3/PGS-neo cells. Bars and error bars represent mean and $S D$, respectively, from three different experiments. (D) The expression of CDX2 and Reg IV and activation of the EGFR/Akt/AP-1 signaling pathway was confirmed by western blot analysis in OMC-3/PGS-CDX2 and OMC-3/PGS-neo cells.

5-FU sensitivity in OMC-3 cells with ectopically overexpressed CDX2. To confirm the effect of CDX2 on 5FU treatment, MTS assay was performed on OMC-3/PGSCDX2 and OMC-3/PGS-neo cells (Figure 4A). 5-FU displayed increased cytotoxicity in OMC-3/PGS-CDX2 cells with an $\mathrm{IC}_{50}(96 \mathrm{~h}) 2.49$ times lower compared to OMC3/PGS-neo cells (Figure 4A). Treatment with 5-FU resulted in inhibition of growth of OMC-3/PGS-neo cells in a dosedependent manner (Figure 4B). Inhibition of growth of OMC-3/PGS-CDX2 cells was stronger (Figure 4B), 


\section{A MTS assay}

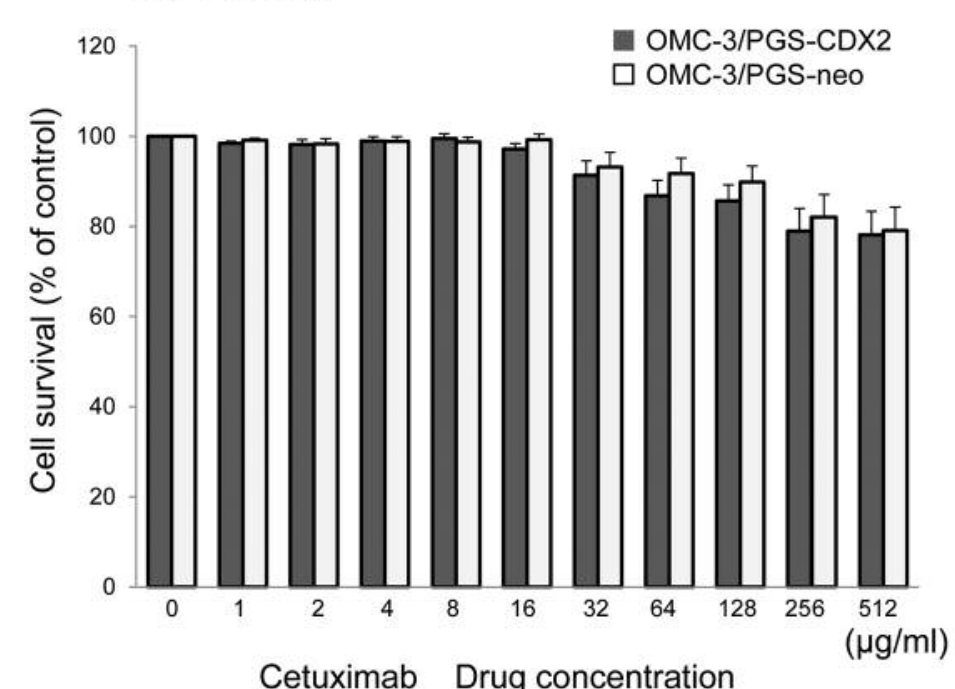

Cetuximab Drug concentration

B
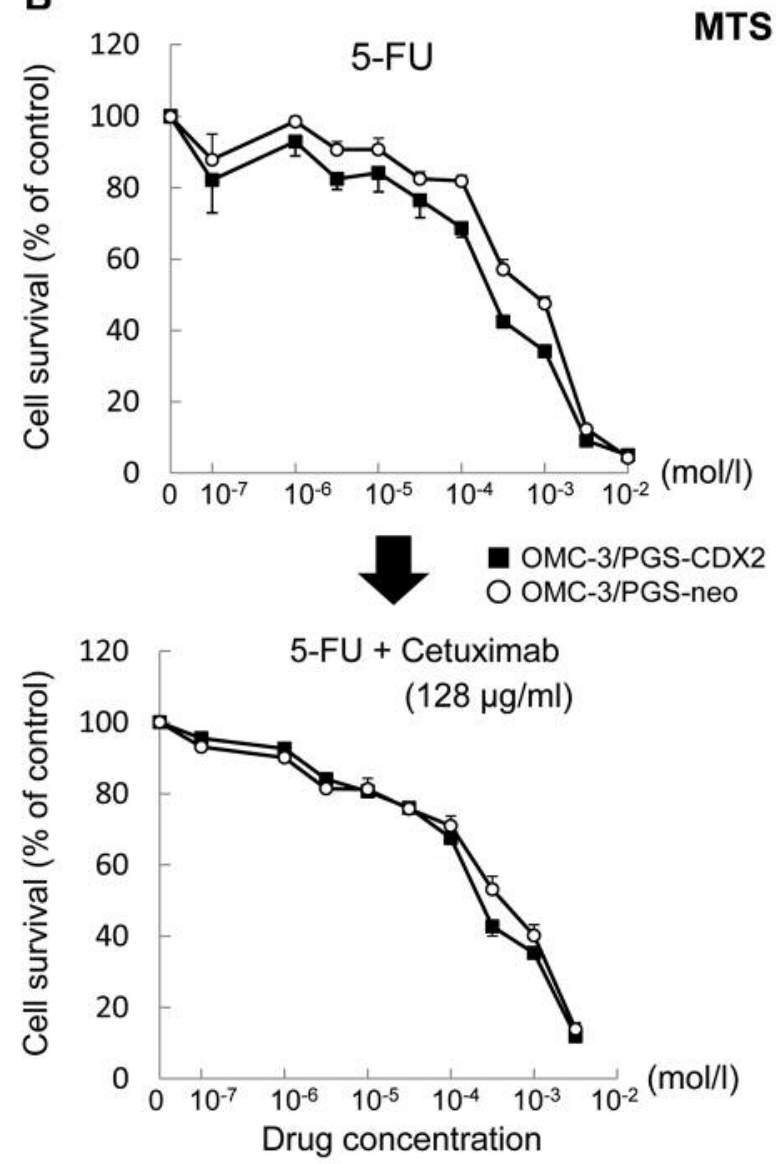
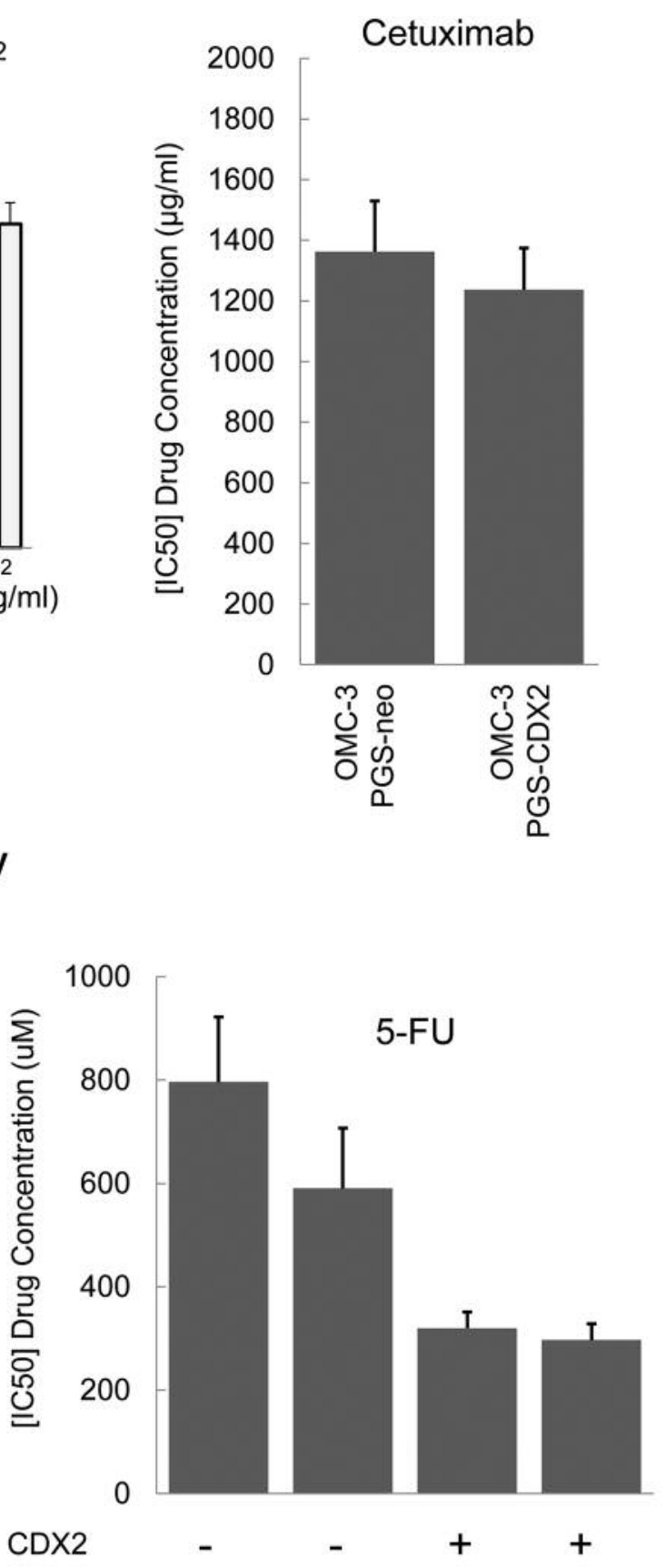

Cetuximab $128 \mu \mathrm{g} / \mathrm{ml}$

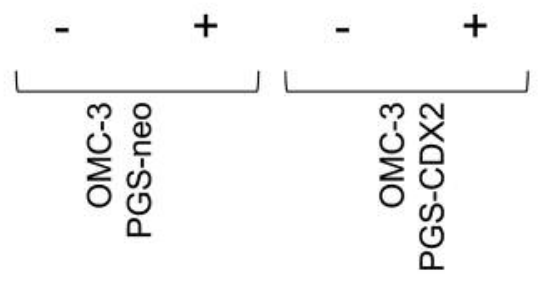

Figure 5. OMC-3 cells ectopically expressing CDX2 display sensitivity to cetuximab. (A) The effect of the anti-EGFR therapeutic antibody cetuximab was poor on ovarian mucinous carcinoma. (B) Combination chemotherapy of 5-FU and cetuximab was poor on ovarian mucinous carcinoma. 
suggesting higher rates of apoptosis in OMC-3/PGS-CDX2 cells. We examined the effects of CDX2 on apoptosis in these cells by forcing its expression. As shown in Figure 4C, OMC-3/PGS-CDX2 cells displayed significantly higher rates of apoptosis in comparison with OMC-3/PGS-neo cells.

Activation of Akt phosphorylation by Reg IV in OMC-3/PGSCDX2 cells. Reg IV induces phosphorylation of EGFR at Tyr992 and Tyr1068, and of Akt at Thr308, which has been shown to increase AP-1 transcription factor activity (18). Bcl-2 is an anti-apoptotic protein located on mitochondria and expressed at high levels in several tumor cells and tissues (23). Expression of Bcl-2 was increased in colon cancer cell lines treated with recombinant Reg IV (18). The phosphorylation of EGFR at Tyr992 and Tyr1068 in OMC3/PGS-CDX2 cells was examined. EGFR was phosphorylated at Tyr992 and Tyr1068 in OMC-3/PGSCDX2 cells, but there was no difference in expression between OMC-3/PGS-CDX2 cells and OMC-3/PGS-neo cells (Figure 4D). The expression of $\mathrm{Bcl}-2$, a protein associated with inhibition of apoptosis, did not change significantly (Figure 4D). These results suggested that CDX2 induced Reg IV expression, but did not induce EGFR phosphorylation and $\mathrm{Bcl}-2$ expression. In addition, we examined phosphorylation of Akt in OMC-3/PGS-CDX2 cells. Akt was phosphorylated in OMC-3/PGS-CDX2 cells (Figure 4D). It has recently been reported that AP-1 induces the expression of dihydropyrimidine dehydrogenase (DPD), the initial rate-limiting enzyme in 5-FU catabolism $(24,25)$. In tumor cell lines, overexpression of DPD is associated with resistance to 5-FU (26). Expression of DPD in OMC-3/PGSCDX2 cells was examined by western blotting and was found to be induced (Figure 4D).

\section{Discussion}

A correlation between the expression of CDX2 and Reg IV proteins and the degree of differentiation of cancer cells in ovarian mucinous carcinoma was observed. Our findings suggest that CDX2 protein regulates the expression of Reg IV in ovarian mucinous carcinoma cell lines, accelerates apoptosis and sensitizes cells to 5-FU. These conclusions are supported by following results.

Overexpression of CDX2 was observed in ovarian mucinous carcinoma, whereas its expression was weak in serous carcinoma, endometrioid carcinoma, and clear cell carcinoma. The expression of CDX2 and Reg IV was most pronounced in well-differentiated mucinous carcinoma and weak in poorly differentiated types. These data suggest that CDX2 and Reg IV expression is associated with the degree of differentiation of cancer cells. Secondly, we investigated the regulation of Reg IV expression by CDX2 using ovarian mucinous carcinoma cell lines. REG4 gene expression and
Reg IV protein expression were enhanced in OMC-3/PGSCDX2 cells. Down-regulation of the expression of CDX2 using RNA interference in OMC-1 cells resulted in reduced expression of the REG4 gene and Reg IV protein. Additionally, we examined EGFR/Akt/AP-1 pathway activation and changes in DPD and Bcl-2 expression by Reg IV expression in ovarian mucinous carcinoma cell lines. In OMC-3/PGS-CDX2 cells, promotion of phosphorylation of EGFR was not seen, but the phosphorylation of Akt was significantly accelerated and enhanced the expression of DPD. Although a decrease in 5-FU sensitivity due to DPD expression has been reported, OMC-3/PGS-CDX2 cells where more sensitive to 5-FU than OMC-3/PGS-neo cells. There was no difference in Bcl-2 expression associated with suppression of apoptosis. However, when an apoptosis assay was performed, apoptosis was promoted in OMC-3/PGSCDX2 cells. Acceleration of apoptosis may have increased 5-FU sensitivity.

CDX2 is overexpressed in the intestinal phenotype of GC and in intestinal metaplasia of the stomach $(27,28)$. In contrast to this, CDX2 expression is lost in poorly differentiated primary colorectal cancer (10). The mechanisms and effects of CDX2 expression in human cancers are largely unknown. Therefore, it is important to clarify the target genes of CDX2. MDR1, which plays an important role in drug resistance in ovarian mucinous carcinoma, has previously been identified as a CDX2 target gene $(7,8,29)$. It was recently discovered that Reg IV proteins potently activate the EGFR/Akt/AP-1 signaling pathway through phosphorylation of EGFR $(18,20)$. High expression of Reg IV in GC cells has been reported to induce Bcl-2 and DPD expression and to suppress apoptosis induced by 5-FU (20). However, in the present study, phosphorylation of EGFR was not induced by expression of Reg IV, and there was also no increase in Bcl-2 expression. There was enhanced apoptosis in ovarian mucinous carcinoma expressing CDX2, despite the enhanced DPD expression and increased sensitivity to 5-FU. The most plausible explanation for these differences is that the level of CDX2-mediated increase in Reg IV is not sufficient enough to induce phosphorylation of EGFR. The relationship between the induction of apoptosis and CDX2 expression is a topic for future investigation.

The current standard chemotherapy protocol for epithelial ovarian cancer is a combination of two drugs: carboplatin and paclitaxel (30). However, this treatment has only a limited anticancer effect in ovarian mucinous carcinoma (26). In our previous study, CDX2 expression in ovarian mucinous carcinoma cells correlated with MDR1 expression, and expression of MDR1 protein induced resistance to chemotherapeutic compounds such as paclitaxel (7). According to the results of this study, the sensitivity of 5-FU was high, but the effect of cetuximab, an anti-EGFR antibody drug, and the effect of the combination 
chemotherapy with 5-FU and cetuximab were poor on ovarian mucinous carcinoma (Figure $5 \mathrm{~A}$ and $\mathrm{B}$ ). Given that CDX2 is a transcription factor that induces differentiation of intestinal epithelial cells, 5-FU, a key chemotherapy drug used for colon cancer, may be a new therapeutic agent in ovarian mucinous carcinoma. In addition to the results of this study, there has also been a report on the confirmed efficacy of the combination chemotherapy of oxaliplatin and 5-FU in cultured cell lines (9).

In conclusion, the results of our study suggest that there is a correlation between the expression of CDX2 and Reg IV in ovarian mucinous carcinoma, and that CDX2 regulates not only the expression of MDR1, but also Reg IV. The reduced sensitivity to paclitaxel due to CDX2 expression may be one of the causes of poor response to combination chemotherapy of paclitaxel and carboplatin, which is the current standard treatment for ovarian mucinous carcinoma. Thus, our study demonstrates the potential of a novel 5-FU-based chemotherapy, based on the expression kinetics of CDX2 in ovarian mucinous carcinoma.

\section{Conflicts of Interest}

The Authors declare no conflicts of interest.

\section{Authors' Contributions}

Iemasa Koh and Yoshiki Kudo designed the study and wrote the initial draft of the manuscript. Iemasa Koh, Suguru Nosaka, Masaki Sekine, Jun Sugimoto and Eiji Hirata contributed to analysis and interpretation of data and assisted in the preparation of the manuscript. Iemasa Koh, Suguru Nosaka, Masaki Sekine, Jun Sugimoto, Eiji Hirata and Yoshiki Kudo have contributed to data collection and interpretation, and critically reviewed the manuscript. All Authors approved the final version of the manuscript and agreed to be accountable for all aspects of the work in ensuring that questions related to the accuracy or integrity of any part of the work are appropriately investigated and resolved.

\section{Acknowledgements}

The Authors thank Dr. Takao Hinoi and Dr. Kazuhiro Sentani for their advices. The Authors also thank the Analysis Center of Life Science, Natural Science Center for Basic Research and Development, Hiroshima University, for use of their facilities.

\section{References}

1 McGuire V, Jesser CA and Whittemore AS: Survival among U.S. women with invasive epithelial ovarian cancer. Gynecol Oncol 84: 399-403, 2002. PMID: 11855877. DOI: 10.1006/ gyno.2001.6536

2 Hess V, A'Hern R, Nasiri N, King DM, Blake PR, Barton DPJ, Shepherd JH, Ind T, Bridges J, Harrington K, Kaye SB and Gore ME: Mucinous epithelial ovarian cancer: a separate entity requiring specific treatment. J Clin Oncol 22: 1040-1044, 2004. PMID: 15020606. DOI: 10.1200/JCO.2004.08.078
3 Cannistra SA: Cancer of the ovary. N Engl J Med 351: 25192529, 2004. PMID: 15590954. DOI: 10.1056/NEJMra041842

4 Winter WE 3rd, Maxwell GL, Tian C, Carlson JW, Ozols RF, Rose PG, Markman M, Armstrong DK, Muggia F and McGuire WP: Prognostic factors for stage III epithelial ovarian cancer: A Gynecologic Oncology Group Study. J Clin Oncol 25: 36213627, 2007. PMID: 17704411. DOI: $10.1200 / J C O .2006 .10 .2517$

5 Pectasides D, Fountzilas G, Aravantinos G, Kalofonos HP, Efstathiou E, Salamalekis E, Farmakis D, Skarlos D, Briasoulis E, Economopoulos T and Dimopoulos MA: Advanced stage mucinous epithelial ovarian cancer: The Hellenic Cooperative Oncology Group experience. Gynecol Oncol 97: 436-441, 2005. PMID: 15863142. DOI: 10.1016/j.ygyno.2004.12.056

6 Pisano C, Greggi S, Tambaro R, Losito S, Iodice F, Maio MD, Ferrari E, Falanga M, Formato R, Iaffaioli VR and Pignata S: Activity of chemotherapy in mucinous epithelial ovarian cancer: a retrospective study. Anticancer Res 25: 3501-3505, 2005. PMID: 16101169.

7 Koh I, Hinoi T, Sentani K, Hirata E, Nosaka S, Niitsu H, Miguchi M, Adachi T, Yasui W, Ohdan H and Kudo Y: Regulation of multidrug resistance 1 expression by CDX2 in ovarian mucinous adenocarcinoma. Cancer Medicine 5(7): 15461555, 2016. PMID: 27060927. DOI: 10.1002/cam4.697

8 Takakura Y, Hinoi T, Oue N, Sasada T, Kawaguchi Y, Okajima M, Akyol A, Fearon ER, Yasui W and Ohdan H: CDX2 regulates multidrug resistance 1 gene expression in malignant intestinal epithelium. Cancer Res 70: 6767-6778, 2010. PMID: 20699370. DOI: 10.1158/0008-5472.CAN-09-4701

9 Sato S, Itamochi H, Kigawa J, Oishi T, Shimada M, Sato S, Naniwa J, Uegaki K, Nonaka M and Terakawa N: Combination chemotherapy of oxaliplatin and 5-fluorouracil may be an effective regimen for mucinous adenocarcinoma of the ovary: A potential treatment strategy. Cancer Sci 100: 546-551, 2009. PMID: 19154404. DOI: 10.1111/j.1349-7006.2008.01065.x

10 Hinoi T, Tani M, Lucas PC, Caca K, Dunn RL, Macri E, Loda M, Appelman HD, Cho KR and Fearon ER: Loss of CDX2 expression and microsatellite instability are prominent features of large cell minimally differentiated carcinomas of the colon. Am J Pathol 159: 2239-2248, 2001. PMID: 11733373. DOI: 10.1016/S0002-9440(10)63074-X

11 Silberg DG, Swain GP, Suh ER and Traber PG: Cdx1 and cdx2 expression during intestinal development. Gastroenterology 119: 961-971, 2000. PMID: 11040183. DOI: 10.1053/gast.2000. 18142

12 Beck F: The role of Cdx genes in the mammalian gut. Gut 53: 1394-1396, 2004. PMID: 15361482. DOI: 10.1136/ gut.2003.038240

13 Hinoi T, Loda M and Fearon ER: Silencing of CDX2 expression in colon cancer via a dominant repression pathway. J Biol Chem 278: 44608-44616, 2003. PMID: 12947088. DOI: 10.1074/ jbc.M307435200

14 Hinoi T, Lucas PC, Kuick R, Hanash S, Cho KR and Fearon ER: CDX2 regulates liver intestine-cadherin expression in normal and malignant colon epithelium and intestinal metaplasia. Gastroenterology 123: 1565-1577, 2002. PMID: 12404231. DOI: 10.1053 /gast.2002.36598

15 Naito Y, Oue N, Hinoi T, Sakamoto N, Sentani K, Ohdan H, Yanagihara K, Sasaki H and Yasui W: Reg IV is a direct target of intestinal transcriptional factor CDX2 in gastric cancer. PLoS ONE 7(11): e47545, 2012. PMID: 23133598. DOI: 10.1371/ journal.pone. 0047545 
16 Hartupee JC, Zhang H, Bonaldo MF, Soares $\mathrm{MB}$ and Dieckgraefe BK: Isolation and characterization of a cDNA encoding a novel member of the human regenerating protein family: Reg IV. Biochim Biophys Acta 1518: 287-293, 2001. PMID: 11311942. DOI: 10.1016/s0167-4781(00)00284-0

17 Chen S, Gou WF, Zhao S, Niu ZF, Zhao Y, Takano Y and Zheng HC: The role of the REG4 gene and its encoding product in ovarian epithelial carcinoma. BMC Cancer 15: 471-482, 2015. PMID: 26077911. DOI: 10.1186/s12885-015-1435-2

18 Bishnupuri KS, Luo Q, Murmu N, Houchen CW, Anant S and Dieckqraefe BK: Reg IV activates the epidermal growth factor receptor/Akt/AP-1 signaling pathway in colon adenocarcinomas. Gastroenterology 130: 137-149, 2006. PMID: 16401477. DOI: $10.1053 /$ j.gastro.2005.10.001

19 Mitani Y, Oue N, Matsumura S, Yoshida K, Noguchi T, Ito M, Tanaka S, Kuniyasu H, Kamata N and Yasui W: Reg IV is a serum biomarker for gastric cancer patients and predicts response to 5-fluorouracil-based chemotherapy. Oncogene 26: 4383-4393, 2007. PMID: 17237819. DOI: 10.1038/sj.onc. 1210215

20 Katsuno Y, Ehata S, Yashiro M, Yanagihara K, Hirakawa K and Miyazono K: Coordinated expression of REG4 and aldehyde dehydrogenase 1 regulating tumourigenic capacity of diffusetype gastric carcinoma-initiating cells is inhibited by TGF- $\beta$. J Pathol 228: 391-404, 2012. PMID: 22430847. DOI: 10.1002/ path. 4020

21 Yoshida M, Kiyozuka Y, Noda T, Imai S and Ichijo M: Establishment and characterization of human cell line derived from mucinous cystadenocarcinoma of ovary. J Jpn Soc Clin Cytol 32: 1-8, 1993. DOI: $10.5795 /$ jjscc.32.1

22 Hayakawa O, Tsumura N, Koizumi M, Endo T and Kudo R: Establishment and characterization of cell line derived from mucinous cystadenocarcinoma of human ovary. Sapporo Ishi 55: 153-160, 1986. DOI: 10.1111/j.1749-0774.2002.tb00111.x

23 Vander Heiden MG and Thompson CB: Bcl-2 proteins: regulators of apoptosis or of mitochondrial homeostasis? Nat Cell Biol 1: E209-216, 1999. PMID: 10587660. DOI: $10.1038 / 70237$
24 Ukon K, Tanimoto K, Shimokuni T, Noguchi T, Hiyama K, Tsujimoto H, Fukushima M, Toge T and Nishiyama M: Activator protein accelerates dihydropyrimidine dehydrogenase gene transcription in cancer cells. Cancer Res 65: 1055-1062, 2005. PMID: 15705907.

25 Harris BE, Song R, Soong SJ and Diasio RB: Relationship between dihydropyrimidine dehydrogenase activity and plasma 5-fluorouracil levels with evidence for circadian variation of enzyme activity and plasma drug levels in cancer patients receiving 5-fluorouracil by protracted continuous infusion. Cancer Res 50: 197-201, 1990. PMID: 2293556.

26 Takebe N, Zhao SC, Ural AU, Johnson MR, Banerjee D, Diasio RB and Bertino JR: Retroviral transduction of human dihydropyrimidine dehydrogenase cDNA confers resistance to 5-fluorouracil in murine hematopoietic progenitor cells and human $\mathrm{CD}^{2} 4^{+}$-enriched peripheral blood progenitor cells. Cancer Gene Ther 8: 966-973, 2001. PMID: 11781659. DOI: 10.1038/sj.cgt.7700393

27 Tatematsu M, Tsukamoto T and Inada K: Stem cells and gastric cancer: role of gastric and intestinal mixed intestinal metaplasia. Cancer Sci 94: 135-141, 2003. PMID: 12708487. DOI: 10.1111/ j.1349-7006.2003.tb01409.x

28 Almeida R, Silva E, Santos-Silva F, Silberg DG, Wang J, De Bolós $\mathrm{C}$ and David L: Expression of intestine-specific transcription factors, CDX1 and CDX2, in intestinal metaplasia and gastric carcinomas. J Pathol 199: 36-40, 2003. PMID: 12474224. DOI: $10.1002 /$ path.1246

29 Pastan I and Gottesman MM: Multidrug resistance. Annu Rev Med 42: 277-286, 1991. PMID: 2035973. DOI: 10.1146/ annurev.me.42.020191.001425

30 Ozols RF: Treatment goals in ovarian cancer. Int J Gynecol Cancer 15: 3-11, 2005. PMID: 15839952. DOI: 10.1111/j.15251438.2005.15351.x 